\title{
Phenomenology of MaVaN's Models in Reactor Neutrino Data
}

\author{
M. F. Carneiro and P. C. de Holanda \\ Instituto de Física "Gleb Wataghin”, Universidade Estadual de Campinas, UNICAMP, 13083-970 Campinas, SP, Brazil \\ Correspondence should be addressed to M. F. Carneiro; mcarneir@ifi.unicamp.br
}

Received 28 June 2013; Revised 27 August 2013; Accepted 1 October 2013

Academic Editor: Seon-Hee Seo

Copyright (C) 2013 M. F. Carneiro and P. C. de Holanda. This is an open access article distributed under the Creative Commons Attribution License, which permits unrestricted use, distribution, and reproduction in any medium, provided the original work is properly cited.

\begin{abstract}
Mass Varying Neutrinos (MaVaN's) mechanisms were proposed to link the neutrino mass scale with the dark energy density, addressing the coincidence problem. In some scenarios, this mass can present a dependence on the baryonic density felt by neutrinos, creating an effective neutrino mass that depends both on the neutrino and baryonic densities. In this work, we study the phenomenological consequence of MaVaN's scenarios in which the matter density dependence is induced by Yukawa interactions of a light neutral scalar particle which couples to neutrinos and matter. Under the assumption of one mass scale dominance, we perform an analysis of KamLAND neutrino data which depends on 4 parameters: the two standard oscillation parameters, $\Delta m_{0,21}^{2}$ and $\tan ^{2} \theta_{12}$, and two new coefficients which parameterize the environment dependence of neutrino mass. We introduce an Earth's crust model to compute precisely the density in each point along the neutrino trajectory. We show that this new description of density does not affect the analysis with the standard model case. With the MaVaN model, we observe a first order effect in lower density, which leads to an improvement on the data description.
\end{abstract}

\section{Introduction}

In cosmology, dark energy is a hypothetical form of energy which permeates all space and leads to the accelerated expansion of the universe [1]. The current standard model of cosmology has $68 \%$ of the total energy of the universe in dark energy [2]. Regardless of its nature, dark energy must have a strong negative pressure to explain the accelerated expansion of the universe and was initially motivated by evidence from supernovae $[3,4]$ and data from cosmic microwave background $[5,6]$.

The need for a new mysterious dark energy component can be interpreted as an indication for physics beyond the standard model. In the present time, the density of dark matter and dark energy is similar, $\rho_{\mathrm{CDM}} / \rho_{\Lambda} \sim 1 / 3$; however this ratio evolves as a function of the scale factor with $1 / a^{3}$. The fact that today these two quantities are of the same order, although they vary dramatically in the history of the universe, became known as the cosmic coincidence problem. The model that intends to explain the dark energy through a cosmological constant needs very specific initial conditions to achieve the correct values for the dark energy density today, giving us clues that this coincidence can be better explained if dark energy has tracked another components of matter since recombination.

Relying on the similarity of scales, $\left(2 \times 10^{-3} \mathrm{eV}\right)^{4}$ for dark energy and $\left(10^{-2} \mathrm{eV}\right)^{2}$ for the neutrino mass split, $[7,8]$ propose to explain the nature of dark energy postulating the neutrino mass as a dynamic quantity, depending on the value of a scalar field $\phi$. Considering a very slow variation in the parameters involved, one can take the system as always in equilibrium, with the potential following the minima.

The potential $\phi$ is then considered very flat and its magnitude dependent on the density of cosmological neutrinos. As a result, these mass varying neutrinos (MaVaN's) become heavier with the decrease of its density. The total energy of the fluid (contained in the neutrinos and in the field $\phi$ ), identified as dark energy, may vary smoothly while the density of neutrinos decreases.

This would explain not only the origin of dark energy but can also significantly modify the limits of cosmological neutrino mass [7], the relationship between the mass of 
the neutrino and leptogenesis [8], and the expected change in the split of flavors for neutrinos and cosmic background from distant astrophysical sources [9].

It can be shown that interactions of subgravitational force can occur naturally between ordinary matter and the field $\phi$, which can cause the value of $\phi$ to vary from its value in vacuum [10]. This leads to neutrinos with masses dependent on the density of the medium and new effects in the flavor oscillation, motivating the study of phenomenological effects of this type of model on the Sun [11-13], in atmospheric neutrinos $[14,15]$, supernovae $[16,17]$, reactor neutrinos $[18$, 19], and neutrinos propagating on Earth [20].

In this work, we investigate the phenomenology of MaVaN's effects in neutrino oscillation, focusing in reactor neutrino data. By applying a parametrization of the effect already used in another context and with a detailed Earth's crust description, we intend to analyze experimental data with this hypothetical matter-neutrino interaction.

In Section 2, we describe the MaVaN formalism and the particular environment approach used and its effects in neutrino oscillations. In Section 3, we present the results and examine how these modifications can affect the reactor neutrino data. In Section 4, we summarize our conclusions.

\section{Materials and Methods}

2.1. MaVaN's Mechanism and Parametrization. Previous works found limits for the product of the effective neutrinoscalar and matter-scalar Yukawa coupling described in [21]. With the assumption of environment homogeneity, the new physics evoked always plays the role of a subleading effect compared to the standard oscillation scenario.

The aim of this work is to find at least one combination of parameters for the new physics that could lead to an acceptable solution to the neutrino oscillation data where such new physics is more than a sub-leading effect. This implies a nonhomogeneity effect of the Earth's crust in the neutrino evolution.

We consider an effective low energy model containing the standard model particles plus a light scalar $(\phi)$ of mass $m_{\phi}$ which couples very weakly both to neutrinos $\left(v_{i}\right)$ and the matter fields $f=e, n, p$.

The Lagrangian takes the form

$$
\begin{aligned}
\mathscr{L}= & \sum_{i} \bar{\nu}_{i}\left(i \not \partial-m_{i}^{0}\right) \nu_{i}+\sum_{f} \bar{f}\left(i \not \partial-m_{f}^{0}\right) f \\
& +\frac{1}{2}\left[\phi\left(\partial^{2}-m_{\phi}^{2}\right) \phi\right]+\sum_{i j} \lambda_{i j}^{v} \bar{\nu}_{i} \nu_{j} \phi+\sum_{f} \lambda^{f} \bar{f} f \phi,
\end{aligned}
$$

where $m_{i}^{0}$ are the vacuum mass that the neutrinos would have in the presence of the cosmic neutrino background and $\lambda_{i j}^{v}$ and $\lambda^{f}$ are, respectively, the effective neutrino-scalar and matter-scalar couplings. We have written a Lagrangian for Dirac neutrinos but equivalently it could be written for Majorana neutrinos.

It has been argued in [22] that, generically, these models contain a catastrophic instability which occurs when neutrinos become nonrelativistic. Since this transition occurs later for hierarchical neutrinos, in what follows we assume the vacuum neutrino masses to be hierarchical:

$$
m_{1}^{0}<m_{2}^{0}<m_{3}^{0}
$$

For solar neutrinos of hierarchical masses, the dominant contribution to the neutrino mass is due to the matter background density. Correspondingly, we neglect the contribution to the neutrino mass from the background neutrino density, and we concentrate on the matter density dependence.

With the additional freedom that the new matter density dependence provides, there is no reason to believe that the three neutrino oscillation dynamics factorize into the dynamics of two neutrino subsystems. However, we will assume that this is still the case and study their effect on solar and KamLAND oscillations under the hypothesis of one mass-scale dominance. Under this assumption, we parametrize the evolution equation as

$$
\begin{aligned}
i \frac{d}{d r}\left(\begin{array}{c}
v_{e} \\
v_{\mu}
\end{array}\right)= & \frac{1}{2 E_{v}} U_{\theta_{12}} \\
& \times\left(\begin{array}{cc}
\left(m_{1}^{0}-M_{1}(r)\right)^{2} & M_{3}^{2}(r) \\
M_{3}^{2}(r) & \left(m_{2}^{0}-M_{2}(r)\right)^{2}
\end{array}\right) U_{\theta_{12}^{\dagger}} \\
& +\left.\left(\begin{array}{cc}
V_{\mathrm{CC}}(r) & 0 \\
0 & 0
\end{array}\right)\left(\begin{array}{c}
v_{e} \\
v_{\mu}
\end{array}\right)\right|_{r=0},
\end{aligned}
$$

where $U_{\theta_{12}}$ is the $2 \times 2$ mixing matrix in vacuum parameterized by the angle $\theta_{12}$ and $M_{i}(r)$ are the environment dependence contributions to the neutrino masses; meanwhile $V_{\mathrm{CC}}(r)=$ $\sqrt{2} G_{F} n_{e}(r)$ is the MSW potential proportional to the electron number density $n_{e}(r)$ in the medium.

The environment effect is introduced as a dependence of the mass terms with the baryonic matter density with the following parametrization:

$$
M_{i}(r)=M_{0 i} \tanh \left[\lambda_{i} \frac{\rho(r)}{\left(\mathrm{g} / \mathrm{cm}^{3}\right)}\right],
$$

which has been chosen to reproduce two features that we intend our mass matrix to present a linear growth of mass with baryonic density for small values of this density as suggested in [21] and a saturation of the environmental dependence of neutrino masses for large values of the baryonic density. It is important to stress that such behaviour is an ad hoc choice of our parametrization in a phenomenological approach to the problem, and it is not derived from details of the interaction between neutrinos, baryonic matter, and the scalar field.

2.2. A Study of the Environment. The KamLAND collaboration uses the constant density crust approximation of $2.7 \mathrm{gr} / \mathrm{cm}^{3}$ in the neutrino evolution analysis. In order to see a more detailed effect than that found in the general literature, we use a specific model of the Earth's crust. The model used was the CRUST 2.0 [23] which is specified in a grid of $2 \times 2$ degrees on the terrestrial surface. 


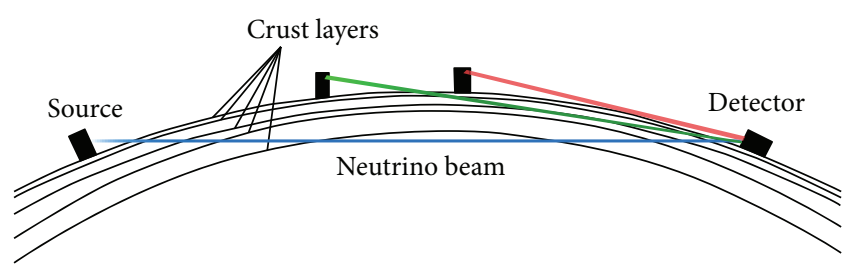

FIGURE 1: Simplified representation of the creation-detection neutrino path, different cases generate different density maps.

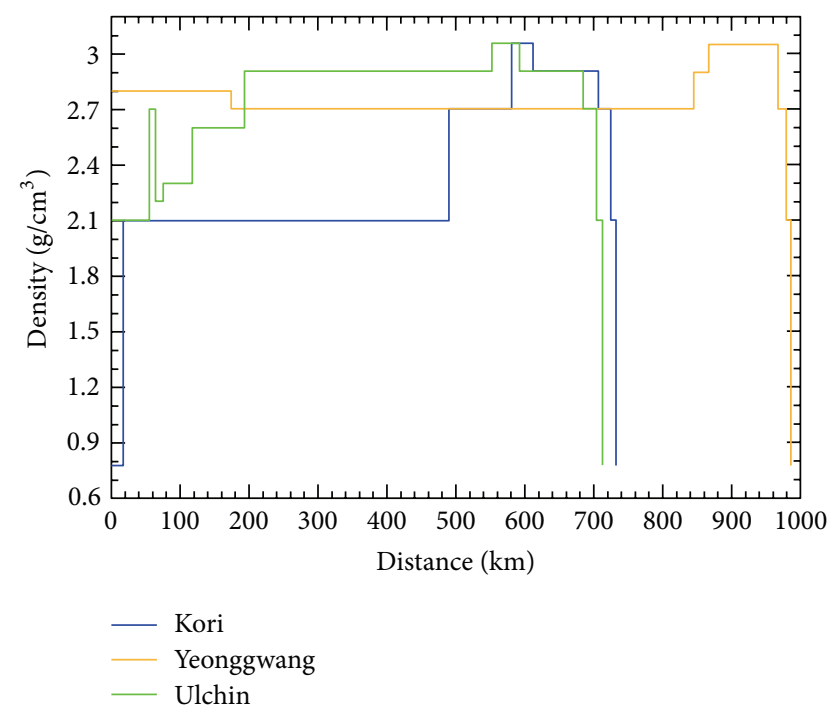

Figure 2: Real density maps used for specific sources, presented in intervals of constant density. Path between the Korean reactors and the detector.

The $2 \times 2$ degree model is composed of 360 key $1 \mathrm{~d}$-profiles where one of these profiles is assigned to each $2 \times 2$ degree cell. Each individual profile is a one-dimensional description of 7 layers: ice, water, soft sediments, hard sediments, upper crust, middle crust, and lower crust. For each of these layers, thickness and density are locally given. Any path determined to be above the ice is considered outside the crust and therefore under the influence of typical atmosphere density.

The KamLAND experiment uses as a source of antineutrinos several different reactors. With the geographic location of each reactor and considering a linear path to the detector, this path will describe a density map specific for each source. The neutrino cross specific values of depth as shown in Figure 1, and hence different densities. Examples are shown in Figure 2. To the best knowledge of the authors of this work this model has not been used before in this context in the reactor neutrinos literature.

2.3. Effects in Reactor Neutrinos Oscillations. As we can see in the specific density maps, it is clear that the description leads to a completely nonadiabatic evolution of the neutrino. So the effective angle variation relative to the local density change causes the nondiagonal terms in the evolution equation of the mass eigenstates to become relevant. So one cannot use here the analytic survival probability.
We use the analysis of the probability amplitudes, which allows local calculation for each region of constant density. Using what is called slab approximation in [24], the effective massive neutrinos propagate as plane waves in regions of constant density.

The transition amplitude $A$

$$
A=\int-i H_{\mathrm{eff}} d r
$$

will be defined in each slab of constant density so $\nu_{1}=$ $\left.A_{0} v_{0}\right|_{r=0}$

$$
A_{\text {total }}=A_{n}, \ldots, A_{1} A_{0}\left|v_{0}\right\rangle=A_{n}, \ldots, A_{1}\left|v_{1}\right\rangle
$$

The notation $A_{n}$ indicates that all the matter-dependent quantities in the $A$ definition must be evaluated with the matter density in the $n$th slab. The final amplitude of a constant density slab is used as initial value at the beginning of the next slab.

In the case of choice, we consider a pure electronic initial neutrino so $\left.v_{0}\right|_{r=0}=\left.\left(\begin{array}{l}1 \\ 0\end{array}\right)\right|_{0}$. In the two families dynamics we have

$$
P_{\bar{\nu}_{e} \bar{\nu}_{e}}=1-\left|A_{21}\right|^{2}
$$

as the survival probability where $A_{21}$ is the final transition amplitude between the point of creation and detection.

We could have a smooth description of the density profiles based on the stepped profiles generated by the model. However, this would result in a calculation much more expensive computationally since integration would be numerical outside the approximation of (6).

2.4. $\chi^{2}$ Analysis. The results of the simulation, that is, the expected number of events for each energy range are analyzed by the method of maximum likelihood. Particularly, the Poisson statistics where the $\chi^{2}$ to be minimized is defined by

$$
\chi^{2} \equiv \sum_{j}^{n} 2\left[K N_{j}^{\mathrm{teo}}-N_{j}^{\mathrm{obs}}+N_{j}^{\mathrm{obs}} \ln \left(\frac{N_{j}^{\mathrm{obs}}}{K N_{j}^{\mathrm{teo}}}\right)\right] \text {, }
$$

where $K$ is a parameter that can vary, leading to a theoretical flux free normalization. The sum is made on the $n$ energy intervals, $N_{i}^{\text {teo }}$ is the event number expected theoretically, and $N_{j}^{\text {obs }}$ is the number of observed events given by collaboration, both in the energy interval $j$.

\section{Results and Discussion}

We developed a code to simulate the KamLAND neutrino events and, based on the presented parametrization and phenomenology of MaVaN's models, we tested the new 
physics. We implemented the model with normal hierarchy and a lighter neutrino with zero mass, to avoid the instability already mentioned:

$$
0=M_{1}=M_{3}=m_{1}<m_{2}<m_{3},
$$

that is, leading to an effect only on the diagonal mass eigenstates

$$
\begin{aligned}
H_{\text {eff }}= & \frac{1}{2 E_{v}} U_{\theta_{12}}\left(\begin{array}{ll}
0 & 0 \\
0 & \left(m_{2}-M_{2}(r)\right)^{2}
\end{array}\right) U_{\theta_{12}}^{\dagger} \\
& +\left(\begin{array}{cc}
V_{C C}(r) & 0 \\
0 & 0
\end{array}\right) .
\end{aligned}
$$

Therefore, the probability amplitude for each interval of constant density can be written as

$$
A_{f}=\left(\begin{array}{rr}
\cos \left(\frac{\Delta m_{f, \mathrm{KL}}^{2}}{4 E_{v}} L_{f}\right)+i \cos 2 \theta_{21}^{m} \sin \left(\frac{\Delta m_{f, \mathrm{KL}}^{2}}{4 E_{v}} L_{f}\right) & -i \sin 2 \theta_{21}^{m} \sin \left(\frac{\Delta m_{f, \mathrm{KL}}^{2}}{4 E_{v}} L_{f}\right) \\
-i \sin 2 \theta_{21}^{m} \sin \left(\frac{\Delta m_{f, \mathrm{KL}}^{2}}{4 E_{v}} L_{f}\right) & \cos \left(\frac{\Delta m_{\mathrm{KL}}^{2}}{4 E_{v}} L_{f}\right)-i \cos 2 \theta_{21}^{m} \sin \left(\frac{\Delta m_{f, \mathrm{KL}}^{2}}{4 E_{v}} L_{f}\right)
\end{array}\right),
$$

where $L_{f}$ is the size of the interval $f, E_{v}$ is the creation energy of the neutrino, and $\cos 2 \theta_{f, 12}^{m}$ is given by

$$
\cos 2 \theta_{f, 12}^{m}=\frac{\Delta \widetilde{M}_{21}^{2}\left(r_{f}\right) \cos 2 \widetilde{\theta}_{f, 12}-2 E_{\nu} V_{\mathrm{CC}}\left(r_{f}\right)}{\Delta m_{f, \mathrm{KL}}^{2}}
$$

The effective KamLAND mass split is given by

$$
\begin{aligned}
\left(\Delta m_{f, \mathrm{KL}}^{2}\right)^{2}= & \left(\Delta \widetilde{M}_{21}^{2}\left(r_{f}\right) \cos 2 \theta_{12}-A_{\mathrm{CC}}\left(r_{f}\right)\right)^{2} \\
& +\left(\Delta \widetilde{M}_{21}^{2}\left(r_{f}\right) \sin 2 \theta_{12}\right)^{2}
\end{aligned}
$$

with $\Delta \widetilde{M}_{21}^{2}\left(r_{f}\right)=\left(m_{2}-M_{2}\left(r_{f}\right)\right)^{2}$ and

$$
M_{2}\left(r_{f}\right)=M_{02} \tanh \left(\lambda_{2} \frac{\rho\left(r_{f}\right)}{\mathrm{g} / \mathrm{cm}^{3}}\right),
$$

where $\rho\left(r_{f}\right)$ is specific density of the interval.

As we can see in (10), we included an effect that does not generate oscillations in the mass eigenstates adding two free parameters that does not interfere with the kinetic term evolution of the neutrino. between

To cover then the parameter space, we let the values run

$$
\begin{aligned}
0.2 & <\tan ^{2} \theta_{12}<0.8 \\
6.0 \times 10^{-5} \mathrm{eV}^{2} & <\Delta m_{21}^{2}<1.0 \times 10^{-4} \mathrm{eV}^{2} \\
0 & <\left|\lambda_{2}\right|<10 \\
-10^{-1} \mathrm{eV} & <M_{02}<10^{-1} \mathrm{eV} .
\end{aligned}
$$

We find the best fit point

$$
\begin{aligned}
\tan ^{2} \theta_{12} & =0.448_{-0.058}^{+0.072}, \\
\Delta m_{21}^{2} & =8.8_{-0.2}^{+0.1} \times 10^{-5} \mathrm{eV}^{2}, \\
\lambda_{2} & =1.258_{-0.108}^{+0.051}, \\
M_{02} & =1.90_{-0.01}^{+0.01} \times 10^{-2} \mathrm{eV}, \\
\frac{\chi_{\min }^{2}}{\text { d.o.f. }} & =\frac{20.10}{15} \longrightarrow \frac{\Delta \chi^{2}}{\Delta \text { d.o.f. }}=\frac{6.22}{2},
\end{aligned}
$$

with

$$
\begin{aligned}
\Delta \chi^{2} & =\chi_{\text {standard }}^{2}-\chi_{\mathrm{MaVaN}}^{2} \\
\Delta \text { d.o.f. } & =\text { d.o.f. } \\
\text { standard } & - \text { d.o.f. } \text { MaVaN }
\end{aligned}
$$

Figures 4-7 show the simulation result and its relation with the values previously obtained from the standard model. The description of the data is not improved in any specific bin. There is an improved description of the data in the general distribution of energy, as we can see in Figure 5. Figures 6 and 7 show, respectively, $\Delta \chi^{2}$ as a function of $\Delta m^{2}$ and $\Delta \chi^{2}$ as a function of $\tan \theta$ with the other parameters fixed in the best fit point given by (16).

We can see that there is a statistically significant improvement of the fit with the inclusion of terms MaVaN; however, we must be careful when interpreting this result, since we choose a specific parametrization on the behaviour of the MaVaN effects that is expected to at least reproduce the quality of the fit from the standard oscillation mechanism.

The value of $M_{2}$ is comparable to $m_{2}^{0}$ for certain values of density and influences directly the effective mass of the neutrino. It is not a second order effect. Indeed $M_{2} \rightarrow m_{2}^{0}$ for $\rho \sim 0.4 \mathrm{~g} / \mathrm{cm}^{3}$ so the difference in effective mass in matter $\Delta \widetilde{M}_{21}^{2}\left(r_{f}\right)$ varies widely for neutrinos crossing density areas with this feature. This kind of resonance creates, in this case, a considerably better description of the data, as can be seen in Figure 3.

Such effect is observed only in a small island in parameter space, as seen in Figure 8. When any one of two parameters 


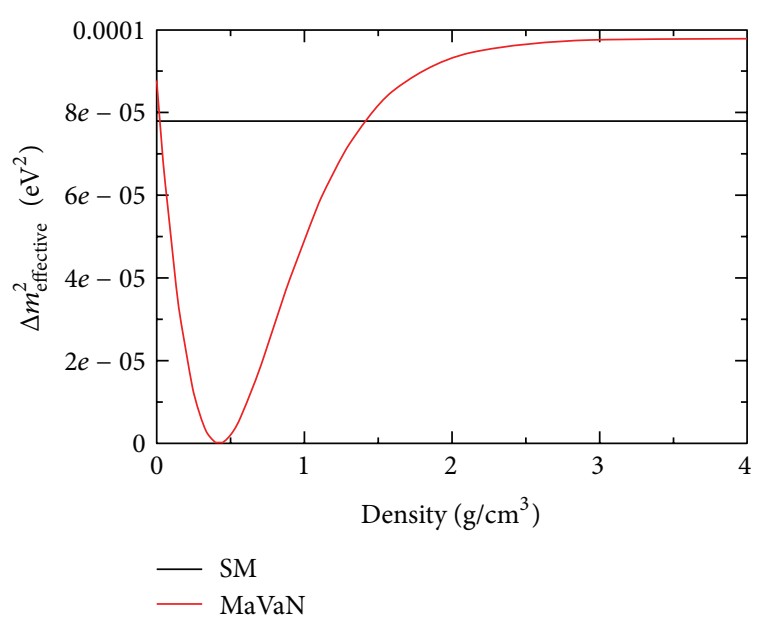

FIGURE 3: Correlation between the effective value of mass after the inclusion of the effect MaVaN and the same term with only the parameters of the standard model.

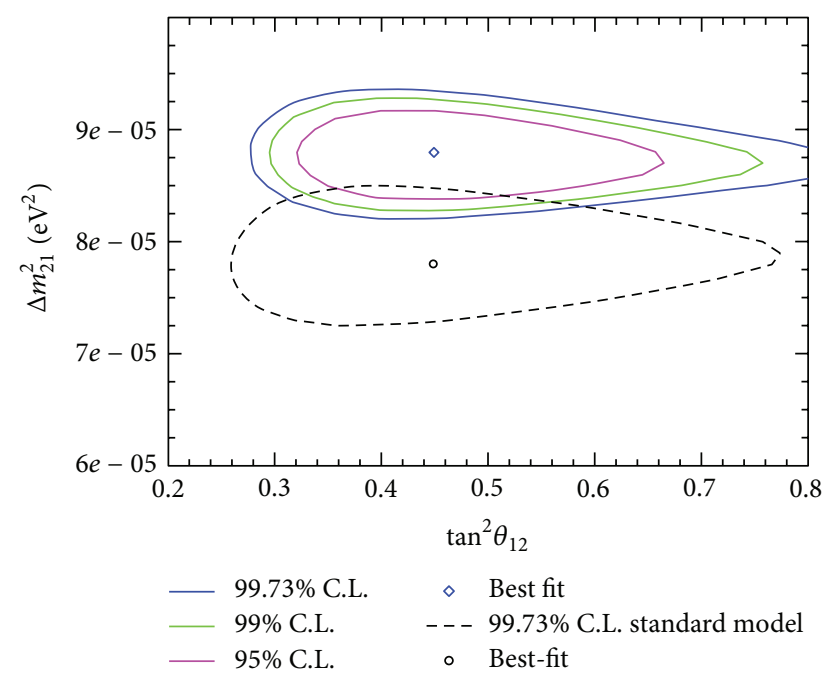

Figure 4: Result of the test for MaVaN model (color curves) compared with simulation data for the standard model (dotted line).

tends to zero, the effect MaVaN also vanishes and returns to the standard model, which is not excluded by our results within $99 \%$ confidence level. A degeneracy occurs because the new parameters tend to compensate each other. We cannot write specific limits for the new physics does to this degeneracy.

In order to quantify the features of this effect inside such island on MaVaN's parameters, we display the global dependence of $\chi^{2}$ on $\lambda_{2}\left(M_{02}\right)$ in Figure 9 (Figure 10) after the marginalization over $\Delta m_{21}^{2}, \tan ^{2} \theta_{12}$, and $M_{02}\left(\lambda_{2}\right)$. From the results we found the following bounds for $90 \%$ and $99.73 \%$ CL:

$$
1.18<\lambda_{2}<1.42 \text {, }
$$

$$
1.89 \times 10^{-2} \mathrm{eV}<M_{02}<1.92 \times 10^{-2} \mathrm{eV} \text {, }
$$

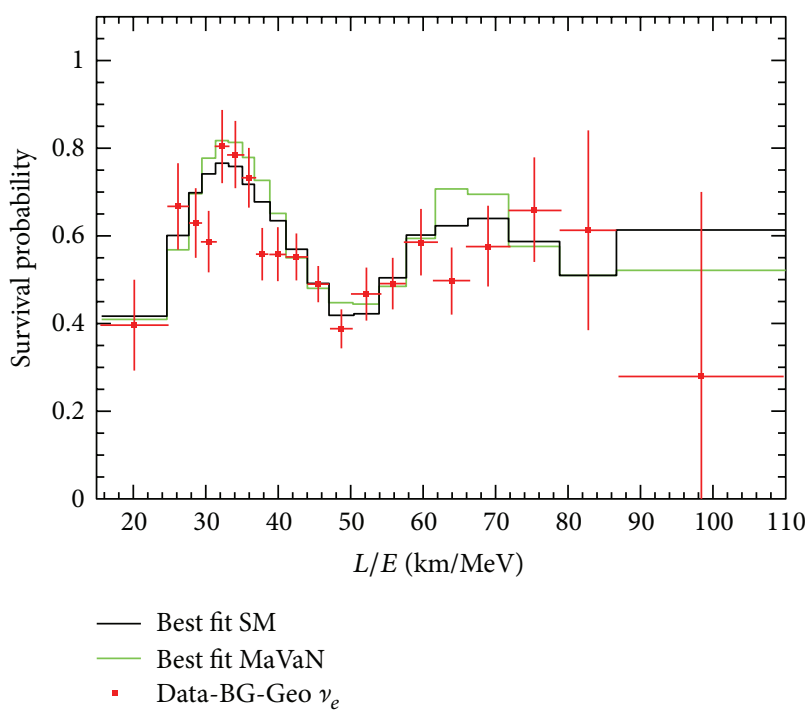

FIGURE 5: Ratio curve of the survival probability of survival with the case of no oscillation. We compared the experimental data (red) with the description of the standard model (black) and the obtained for the MaVaN model (green).

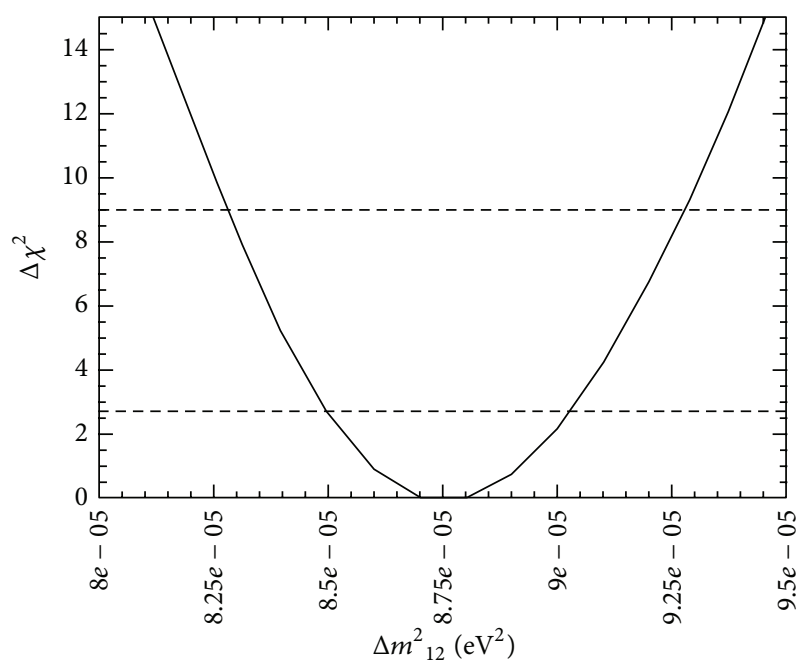

FIGURE 6: $\Delta \chi^{2}$ as a function of $\Delta m^{2}$, when the other parameters are fixed in the best fit point, given by (16). Dotted lines indicate, from bottom to top, $90 \%$ and $99.73 \%$ confidence levels.

$$
\begin{gathered}
1.15<\lambda_{2}<1.60, \\
1.88 \times 10^{-2} \mathrm{eV}<M_{02}<1.93 \times 10^{-2} \mathrm{eV},
\end{gathered}
$$

taking into account, for these specific limits, only the island of best fit. The standard model would be included in the intervals of $99 \%$ confidence level if we take into account the whole parameter space.

The results and analysis presented here must be viewed as a specific choice of a particular model. We have three sources of arbitrariness in the model, the choice of $m_{1}=M_{1}=0$ as $M_{3}=0$ and the hypothesis of the hyperbolic tangent 


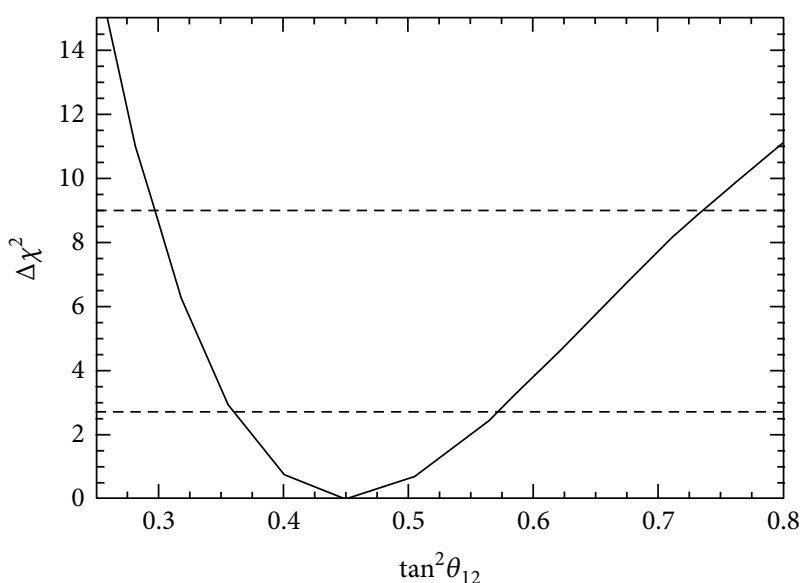

FIGURE 7: $\Delta \chi^{2}$ as a function of $\tan \theta$, when the other parameters are fixed in the best fit point, given by (16). Dotted lines indicate, from bottom to top, $90 \%$ and $99.73 \%$ confidence levels.

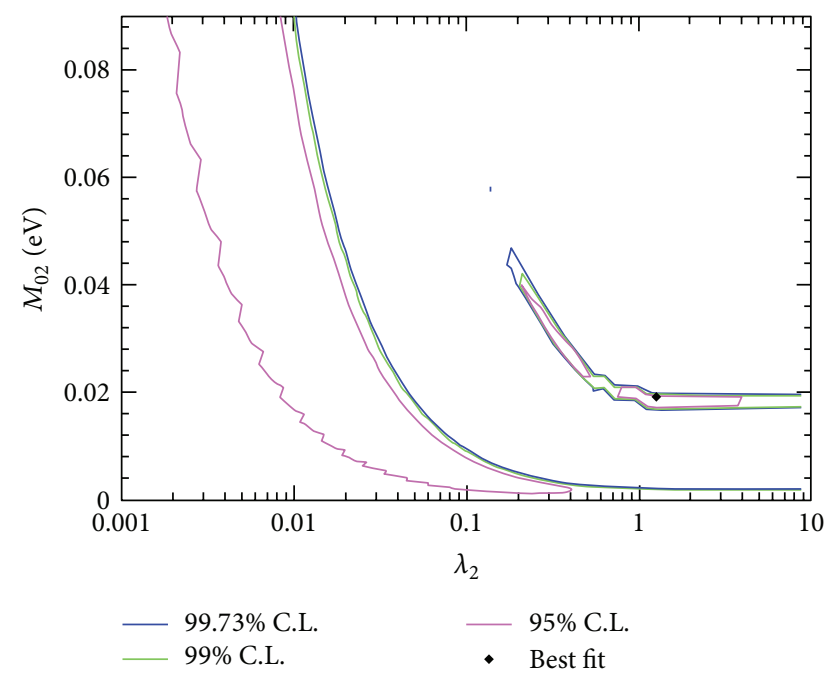

FIGURE 8: Exclusion curves in the parameter space MaVaN to $95 \%$, $99 \%$, and $99.73 \%$ confidence levels. We can see that the effect appears only in an island of values and does not exclude the standard model, which becomes dominant when the parameters tend to zero.

parametrization. However, we expect that similar effects would be present if we change any of these assumptions.

\section{Conclusions}

We studied the phenomenological effects of a specific dependence of the effective neutrino mass with the local density of the propagation medium, considering a model that included the standard model plus a light scalar $(\phi)$ that couples weakly to all constituents of the matter. We used a specific parameterization for the new physics and a detailed description of the Earth's crust density, the CRUST 2.0, which was not previously used in the literature in this context.

Assuming that neutrinos masses follow the hierarchy $0=$ $m_{1}^{0}<m_{2}^{0}<m_{3}^{0}$, we analyzed data from KamLAND (20 points

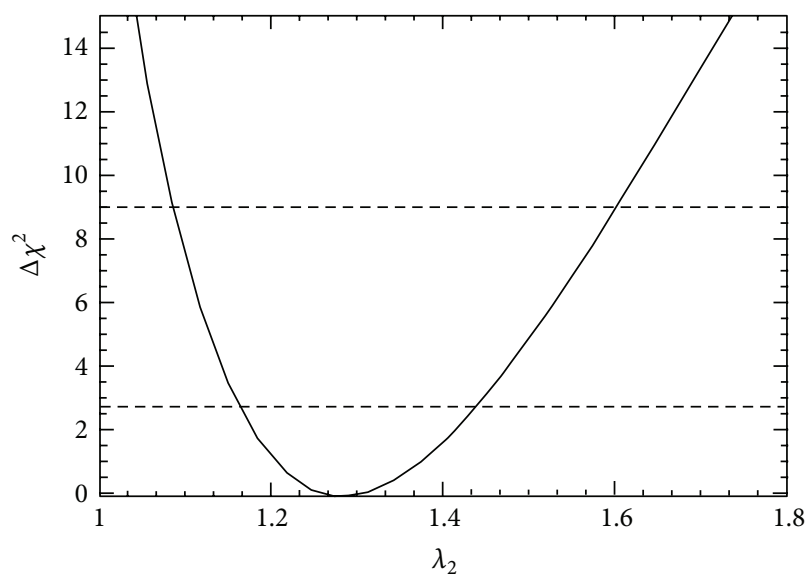

Figure 9: Dependence of $\chi^{2}$ on the free parameter $\lambda_{2}$. Dotted lines indicate $90 \%$ and $99.73 \%$ confidence levels.

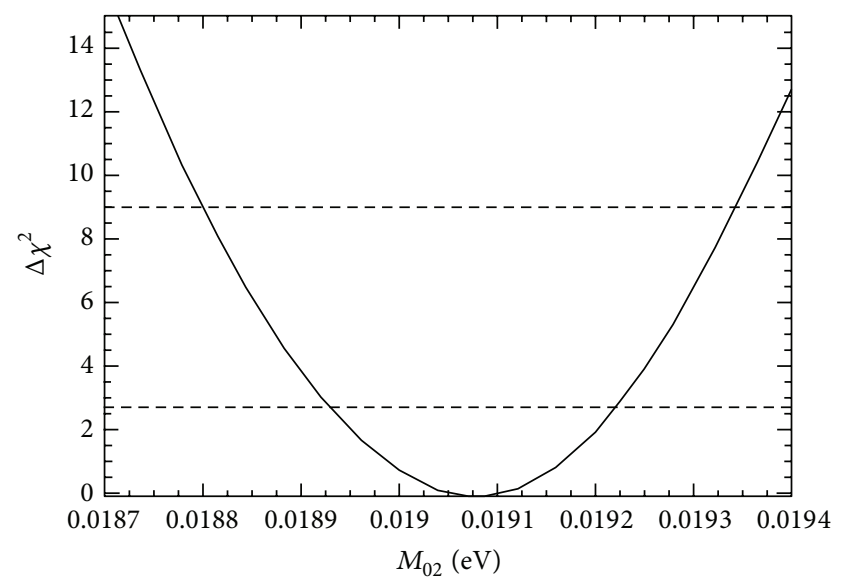

FIgURE 10: Dependence of $\chi^{2}$ on the free parameter $M_{02}$. Dotted lines indicate $90 \%$ and $99.73 \%$ confidence levels.

data) in the context of this effective model. Our analysis depends on four parameters: the two standard oscillation parameters $\Delta m_{21}^{2}=\left(m_{2}^{0}\right)^{2}, \tan ^{2} \theta_{12}$ and the two coefficients related to the MaVaN model, $\lambda_{2}$ and $M_{02}$. We found the best fit and the $68.27 \%$ C.L. in $\tan ^{2} \theta_{12}=0.448_{-0.058}^{+0.072}$, $\Delta m_{21}^{2}=8.8_{-0.2}^{+0.1} \times 10^{-5} \mathrm{eV}^{2}, \lambda_{2}=1.258_{-0.108}^{+0.051}$, and $M_{02}=$ $1.90_{-0.01}^{+0.01} \times 10^{-2} \mathrm{eV}$. This point corresponds to a decrease of $\Delta \chi^{2} / \Delta$ d.o.f. $=6.22 / 2$ compared to the minimum where only the standard matter effect was considered. However, we have seen that this strong effect, although statistically significant, appears in an island of specific values, and the results remain consistent with the standard model within $99 \%$ confidence level.

We observed that the new physics is more then a subleading effect and clearly dependent on the specific density description used. Such effect appears due to a strong variation of neutrino parameters at lower densities than the value usually used as constant for Earth crust in the literature, impliyng a nonhomogeneity effect of the Earth's crust in the neutrino evolution. 
To write specific limits including the null value for the new physics parameters is not possible due to a parameter degeneracy. The standard model is reproduced when any of the parameters that describes the MaVaN effects goes to zero. We believe that a joint analysis with solar neutrinos may clarify this point. These limits will be tested in the future for an analysis with solar neutrinos.

\section{Acknowledgments}

The authors would like to thank Daniel Boriero for his valuable and constructive suggestions during the planning and development of this paper. They also thank CNPq and CAPES for several financial supports.

\section{References}

[1] P. J. E. Peebles and B. Ratra, "The cosmological constant and dark energy," Reviews of Modern Physics, vol. 75, no. 2, pp. 559606, 2003.

[2] P. Ade, N. Aghanim, C. Armitage-Caplan et al., "Planck 2013 results. XVI. Cosmological parameters," http://arxiv.org/abs/ 1303.5076.

[3] A. G. Riess, A. V. Filippenko, P. Challis et al., "Observational evidence from supernovae for an accelerating universe and a cosmological constant," The Astronomical Journal, vol. 116, no. 3, p. 1009, 1998.

[4] S. Perlmutter, G. Aldering, G. Goldhaber et al., "Measurements of $\Omega$ and $\Lambda$ from 42 high-redshift supernovae," The Astrophysical Journal, vol. 517, no. 2565, 1999.

[5] P. de Bernardis, P. A. R. Ade, J. J. Bock et al., "A flat Universe from high-resolution maps of the cosmic microwave background radiation," Nature, vol. 404, p. 955, 2000.

[6] C. Bennett et al., "First-year Wilkinson Microwave Anisotropy Probe (WMAP) observations: preliminary maps and basic results," The Astrophysical Journal Supplement Series, vol. 148, no. 1, p. 1, 2003.

[7] R. Fardon, A. E. Nelson, and N. Weiner, "Dark energy from mass varying neutrinos," Journal of Cosmology and Astroparticle Physics, vol. 2004, no. 10, article 005, 2004.

[8] P. Gu, X. Wang, and X. Zhang, "Dark energy and neutrino mass limits from baryogenesis," Physical Review D, vol. 68, Article ID 087301, 4 pages, 2003.

[9] P. Q. Hung and H. Päs, "Cosmo MSW effect for mass varying neutrinos," Modern Physics Letters A, vol. 20, no. 16, p. 1209, 2005.

[10] N. Weiner and K. M. Zurek, "New matter effects and BBN constraints for mass-varying neutrinos," Physical Review D, vol. 74, no. 2, Article ID 023517, 2006.

[11] M. Cirelli, M. Gonzalez-Garcia, and C. Peña-Garay, "Mass varying neutrinos in the Sun," Nuclear Physics B, vol. 719, no. 1-2, pp. 219-233, 2005.

[12] V. Barger, P. Huber, and D. Marfatia, "Solar mass-varying neutrino oscillations," Physical Review Letters, vol. 95, no. 21, Article ID 211802, 2005.

[13] P. C. de Holanda, "Possible scenario for MaVaN's as the only neutrino flavor conversion mechanism in the Sun," Journal of Cosmology and Astroparticle Physics, vol. 2009, no. 7, article 024, 2009.

[14] D. R. Gratieri and O. L. B. Peres, inpreparation.
[15] K. Abe, Y. Hayato, T. Iida et al., "Search for matter-dependent atmospheric neutrino oscillations in Super-Kamiokande," Physical Review D, vol. 77, Article ID 052001, 6 pages, 2008.

[16] F. Rossi-Torres, M. Guzzo, P. de Holanda, and O. Peres, "Mass varying neutrinos in supernovae," Physical Review D, vol. 84, no. 5, Article ID 053010, 8 pages, 2011.

[17] M. M. Guzzo and F. Rossi-Torres, "Neutrinos de massa variável," Physicee, vol. 9, p. 9, 2010.

[18] M. Gonzalez-Garcia, P. C. de Holanda, and R. Z. Funchal, "Effects of environment dependence of neutrino mass versus solar and reactor neutrino data," Physical Review D, vol. 73, no. 3, Article ID 033008, 9 pages, 2006.

[19] T. Schwetz and W. Winter, "Testing mass-varying neutrinos with reactor experiments," Physics Letters B, vol. 633, no. 4-5, pp. 557-562, 2006.

[20] V. Barger, D. Marfatia, and K. Whisnant, "Confronting massvarying neutrinos with MiniBooNE," Physical Review D, vol. 73, no. 1, Article ID 013005, 10 pages, 2006.

[21] D. B. Kaplan, A. E. Nelson, and N. Weiner, "Neutrino oscillations as a probe of dark energy," Physical Review Letters, vol. 93, no. 9, Article ID 091801, 2004.

[22] N. Afshordi, M. Zaldarriaga, and K. Kohri, "Instability of dark energy with mass-varying neutrinos," Physical Review D, vol. 72, no. 6, Article ID 065024, 10 pages, 2005.

[23] C. Bassin, G. Laske, and G. Masters, "The current limits of resolution for surface wave tomography in North America," Eos, Transactions American Geophysical Union, vol. 81, p. F897, 2000.

[24] C. Giunti and C. W. Kim, Fundamentals of Neutrino Physics and Astrophysics, Oxford University Press, 1st edition, 2007. 

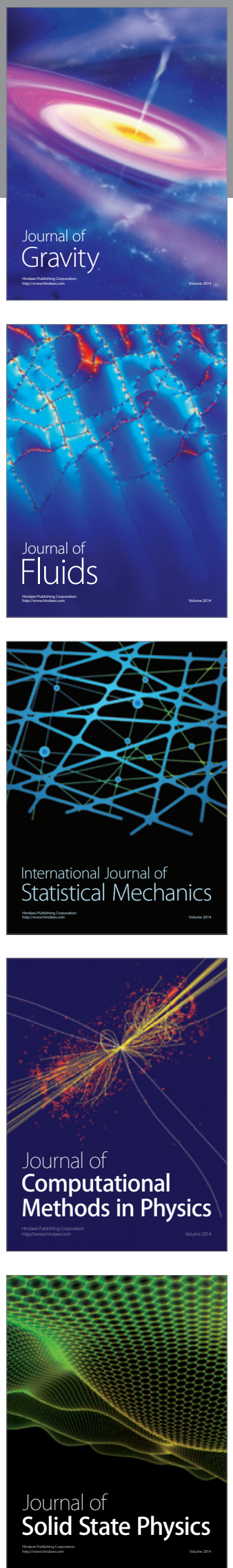

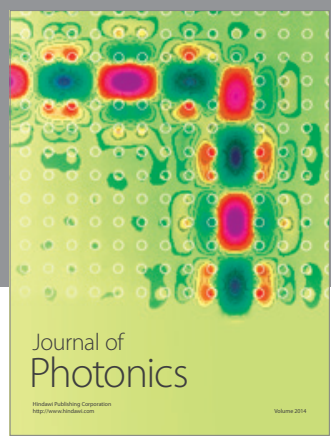

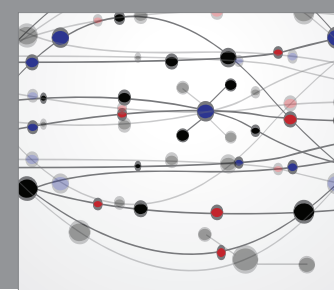

The Scientific World Journal

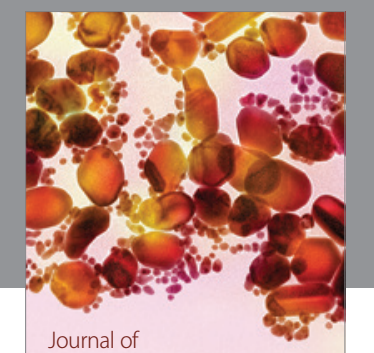

Soft Matter
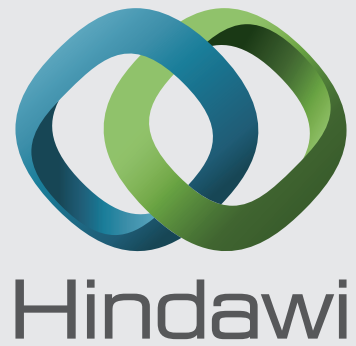

Submit your manuscripts at

http://www.hindawi.com
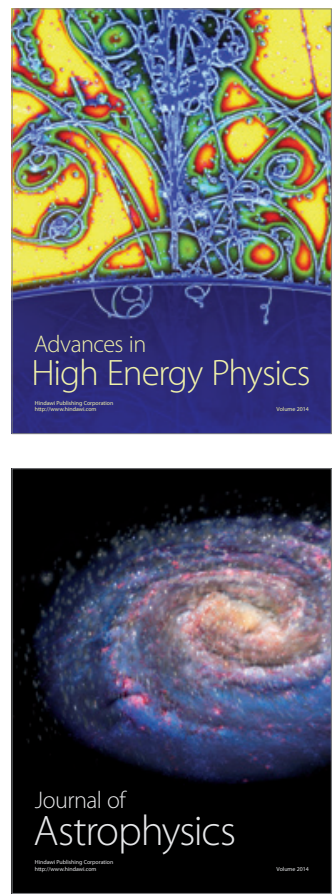
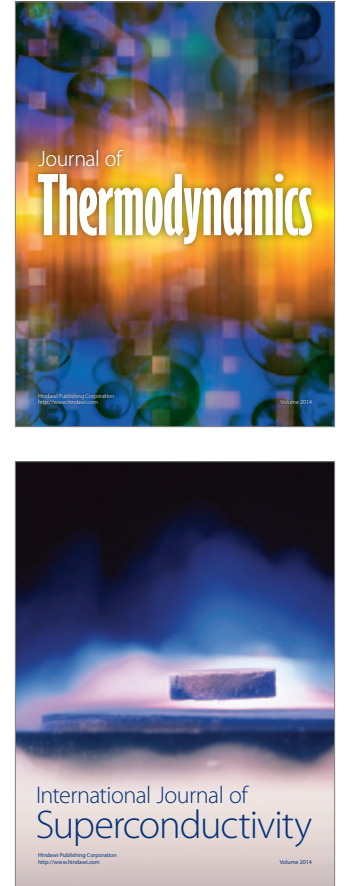
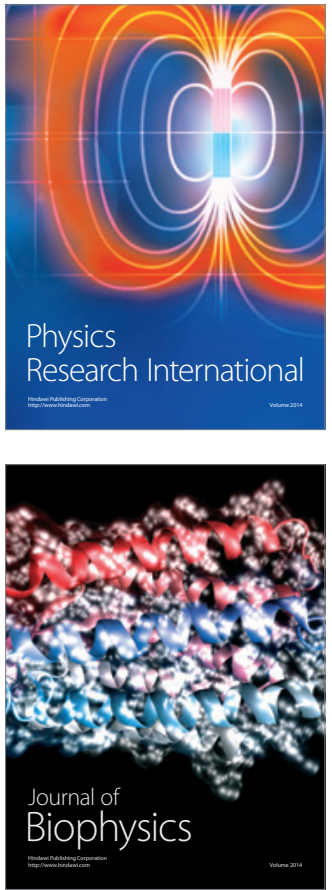
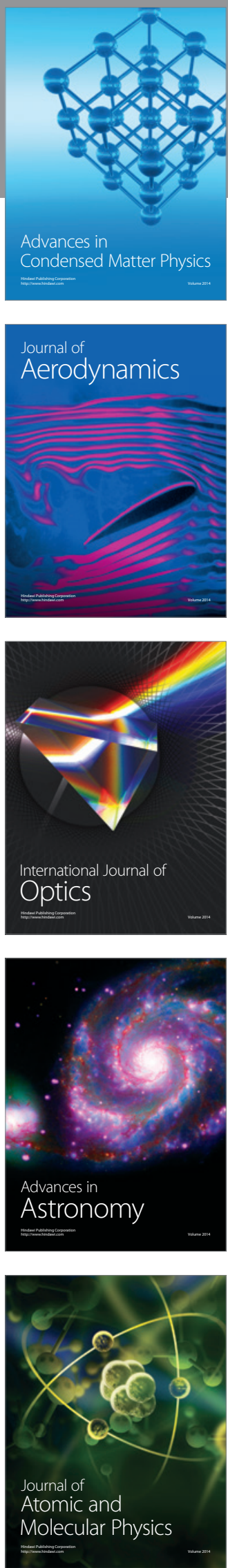